\title{
Safe and Ecological Speed Profile Planning Algorithm for Autonomous Vehicles Using a Parametric Multiobjective Optimization Procedure
}

\author{
Olivier Orfila ${ }^{1} \quad$ Dominique Gruyer ${ }^{\text {1) }}$ Karima Hamdi 1) Sébastien Glaser ${ }^{2)}$ \\ 1) IFSTTAR, COSYS, LIVIC, F-78000 Versailles, France \\ 25 allée des Marronniers Versailles, F-78000, France \\ (E-mail: olivier.orfila@ifsttar.f,r, E-mail: dominique.gruyer@ifsttar.fr, E-mail: karima.hamdi@ifsttar.fr) \\ 2) QUT, CARRS-Q, K Block, 130 Victoria Park Road, Kelvin Grove, QLD 4059, Australia \\ (E-mail: sebastien.glaser@qut.edu.au)
}

Received on December 12, 2017

\begin{abstract}
This paper proposes and evaluates an algorithm called Multi-Objective planning based on Simulated Annealing (MOSA) that plans a trajectory (speed profile) for a passenger car on a free, single lane road. This algorithm is relying on a decomposition of the decision space into "chunks" that are optimized separately. Two objectives have been taken into account: travel time and fuel consumption. Optimization constraints are built from safety modelings combining legal speed, curves speed limits and junctions limits. The multi-objective optimization is performed through a linear scalairisation method and the optimization is a parametric optimization based on simulated annealing.

The algorithm has been tested on simulated annealing convergence and results show a good convergence under 500 iterations and a small sensitivity to variables initialization. However, sensitivity to core parameters of the simulated annealing (initial temperature and temperature decreasing rate) is very high and some guidelines for the calibration of these parameters are given in this paper. Then, the algorithm has been tested and compared to experimental results and it shows that, even if some drivers can drive the road quicker than the algorithm, they cannot drive with a lower fuel consumption. Furthermore, the algorithm results are better than the most of the experimental results according to the Pareto definition of dominance and global results outperform results from another planning algorithm based on Dijkstra's algorithm.

Future works will concentrate on improving the algorithm to be more reactive to unexpected obstacles and more consistant in the “chunks" transitions.
\end{abstract}

KEY WORDS: Electronics and Control, Speed profile optimization, Autonomous vehicle, energy consumption, MOSA [E1]

\section{INTRODUCTION}

The autonomous vehicle is currently on everyone's lips, from the largest automakers/OEM to the newcomers in this field (Google, Tesla ...). Driving automation, once synonymous with science fiction, is now expected as a silver bullet to solve road safety, impact of transport on the environment and congestion of road infrastructures issues. However, main issues related to technology, legislation and ethics still stand in the way of mass marketing. One of the most complex topic concerns the driver acceptance in a safe and green environment.

Autonomous driving is mainly relying on perception, decision, planning and control steps. On one hand, perception, which consists in detecting/assessing/identifying the environment "key components" from a static and dynamic point of view, has been widely researched in last decades. The results and the progresses of these researches can be seen everyday in numerous prototypes (1)(10)(11)(12). Control, consisting in following a determined trajectory, has also been deeply studied in the last decades and it can be considered as a closed issue from the automotive point of view (for standard cars) ${ }^{(2)}$. Decision and planning, on the other hand has become a more and more interesting topic consisting in defining a possible and/or optimal trajectory to be followed on the detected environment $(3,4,13,14,15,16)$. This implies efficient algorithms that computes multiple possibilities. A characteristic that might increase this level of complexity is that the transportation trajectory planning problems are multi-objectives. A car needs to reach its destination as quickly as possible by keeping its occupants in highly safety conditions and consuming the lowest quantitity of energy.

This article proposes and evaluates a speed profile planning algorithm warranting safety, optimizing energy use, and managing travel time of an autonomous vehicle in order to increase the driver and passengers acceptance. This algorithm is based on a parametric description of a speed profile that is then generated using a multiobjective optimization procedure. The parametric description of the speed profile, inspired by traffic simulation modeling has been designed to maximize driver and passengers acceptance. Safety rules and safety parameters are taken into account in the first step of the algorithm where safety constraints are defined. Afterwards, the speed profile is optimized within this generated constraints set.

\section{METHODOLOGY}

This paper proposes and evaluates an algorithm called MultiObjective planning based on Simulated Annealing (MOSA).

The first step of this study is to develop the algorithm. MOSA is able to build a constrained set from road properties and to generate optimal speed profiles according to the Pareto definition of optimality ${ }^{(5)}$. Then, safety is considered as a constraint and both fuel use and travel time are selected as objectives. The main idea of the algorithm is to share/split the speed profile in "chunks", each of them being independent. Each of these chunks is then optimized using a multi-objective strategy (linear scalairisation) ${ }^{(6)}$ and an optimization algorithm (simulated annealing) ${ }^{(7)}$. Finally a set of optimal trajectories is generated. In this step, a detailed 
evaluation of the simulated annealing applied to speed profile optimization is performed. First, the convergence of the algorithm is checked in a condition where the result of the optimization is known. Then, a sensitivity analysis is performed on the initial values of the optimized variables and on two of the main parameters of the simulated annealing algorithm (initial temperature, temperature decreasing rate).

In a second step, the algorithm is evaluated by comparing it to a method from the literature coming from operational research methods (Dijkstra) and by comparing it to experimental results from human drivers. Here, an experimental dataset has been built with 21 participants driving in two conditions (standard and ecodriving) on the same road. All roads parameters have been measured with a dedicated vehicle equipped with embedded sensors. More information will be given in a next section of this paper. This enable us to compare an optimal algorithm to humans trying to drive in an optimal way. The comparison is mainly performed using the definition of the Pareto frontier. This frontier, made of dominant solutions, is also used to compare the algorithms together.

\section{CHUNKED PARAMETRIC MULTIOBJECTIVE OPTIMIZATION}

The proposed algorithm (MOSA) has several properties:

- Chunked: The speed limit profile is split into sub elements (chunks) in order to reduce the computational complexity.

- Multi-objective: Each chunk is processed separately using a multi-objective optimization method. For each chunk, several speed profiles are generated using a linear scalairisation method where a weight is associated to each objective.

- Optimal: An optimization procedure is used to generate and optimize each chunk. Here, a simulated annealing has been used but others multi-dimensionnal optimizations might be used.

- $\quad$ Parametric: On each chunk, the speed profile is defined from an analytic function (a combination of segments based on IDM from M. Treiber $(17,18)$ ) where a set of 5 parameters defines a speed profile of a chunk.

The following part of this section describes the algorithm in details.

\subsection{Generating constraints}

In order to generate speed profiles, the first step consists in the generation of speed constraints. These constraints are coming from safety aspects such as legal speed limits, safe speeds in curves,...). This generation of constraints is done in three stages:

- Collection of main upcoming road data such as road grade, road speed limit, road curvature, junction type, presence of road elements (pedestrian crossing, speed bump...).

- Conversion into speed limits: From the collected data, safety constraints are generated that will limit the optimization research space. This step is performed by modeling safety limits from a simplified vehicle dynamic model (point model with Coulomb friction) combined to a human driver model (IDM) ${ }^{(17)}$ in order to evaluate safe speed limits in curves and junctions. In this last model, a simple acceleration limit, depending on speed has been used.

- Each previously collected data element is then associated to a speed limit and a combination of these different speed limits, by selecting the lowest one, allows to build a general speed limit on the selected route (in red on figure 2).

It can be noticed in Figure 2 that the limit speed profile is made of square signals in order to facilitate the chunking step.

Finally, the parameter vector is optimized several times according to the weights associated to the travel time and energy economy criteria. The variation of these weights defines the driver style. Several optimization methods (genetic algorithm, simulated annealing and simplex search) are then compared during an evaluation step. For a D type speed profile (steady speed), depending on the energy use modeling, the optimization can be done analytically.

\subsection{Chunking}

This speed limit is chuncked into several elementary parts, each of them being constituted of an elementary speed profile that can be one of the four types (A: acceleration and constant speed phase; B: acceleration, steady speed and deceleration, C: steady speed and deceleration, D: steady speed) as presented in figure 1 . The type of speed profile depends on the type of edge on the speed limit. For example, If two falling edges are succeeding then the speed profile is a $\mathrm{C}$ type. If two rising edge are succeeding then the speed profile is a A type. If a rising edge is followed by a falling edge, the speed profile is a B type and if a falling edge is followed by a rising edge, the speed profile is D type. A safety margin has been added to be sure that chunks are not too short because of input signal quality issues. Then a chunk can not be shorter than 20 meters.

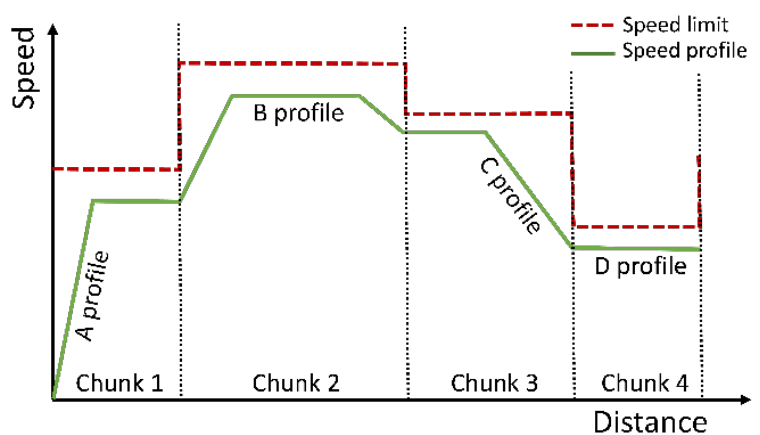

Fig. 1: Chunking methodology description on standards speed profiles

A result of chunking is showed in Figure 2 where a trip of $15 \mathrm{~km}$ has been split into a set of elements. This method will almost have linear computational time consumption when the distance increases. This computational time consumption will be highly influenced by the number and lengths of chunks. 


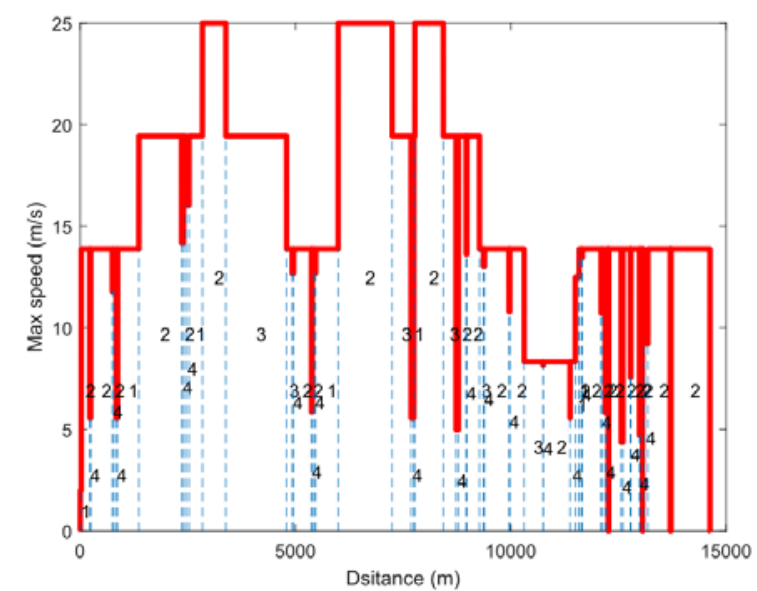

Fig. 2: Result of chunking algorithm displaying speed limit from the constraints generation. Numbers under the speed profiles define the type of chunk from 1 to 4 corresponding to type A to D respectively.

\subsection{Generating a speed profile}

Each elementary speed profile is composed, depending on the chunk type, of three phases:

- A type: Acceleration, linear speed variation and acceleration

- B type: Acceleration, linear speed variation and deceleration

- C type: Deceleration, linear speed variation and deceleration

- D type: Deceleration, linear speed variation and acceleration

Acceleration and deceleration speed profiles are generated with the IDM (Intelligent Driver Model, Treiber, 2000)(17) equations which represent the human driver behavior while accelerating. The free acceleration equation of this model used in this paper is given by:

$$
\dot{v}^{\text {free }}=a\left[1-\left(\frac{v}{v_{0}}\right)^{4}\right]
$$

Where $\dot{v}^{\text {free }}$ is the time derivative of the vehicle velocity, $v_{0}$ the desired velocity, $a$ the maximal longitudinal acceleration, $v$ the vehicle velocity.

It is here supposed that using this function will increase the driver and passengers acceptance of the autonomous vehicle. Acceleration and deceleration curves can be defined by 3 parameters (acceleration at origin, desired speed, final deceleration). The linear speed variation phase is simply an connection interface between the acceleration phase and the deceleration phase. Then, to totally describe the speed profile on one chunk, only five parameters are required:

- $\quad$ a: Initial acceleration $\left(\mathrm{ms}^{-2}\right)$

- $\mathrm{V}_{\text {des }}$ : desired speed $\left(\mathrm{ms}^{-1}\right)$

- $\mathrm{p}_{\mathrm{a}}$ : percentage of distance of acceleration phase

- $\quad \mathrm{p}_{\mathrm{d}}$ : percentage of distance of deceleration phase

- $\quad \mathrm{d}$ : Final deceleration $\left(\mathrm{ms}^{-2}\right)$

\subsection{Multi-objective optimization}

Multiobjective optimization is making a multicritera analysis process to find the best compromise among alternative decisions and optimize a number of possibly conflicting objectives simultaneously. It this work, it is formuled as:

$$
\begin{aligned}
& \min \left\{f_{1}(x), f_{2}(x)\right\} \\
& x \in \Omega,
\end{aligned}
$$

Where: $f_{i}: \mathbb{R}^{n} \rightarrow \mathbb{R}, i=1,2$ are the (conflicting) objectives and $\Omega \subseteq \mathbb{R}^{n}$ is a feasible region. Each of the functions $f_{i}$ represents an objective. In this case, two objectives are considered: Travel time and fuel consumption. As in this paper, we consider a safe and ecological speed profile planning as a multiobjective optimization problem, where the goal is to minimize the energy consumption and the the travel time.

Several ways exist to perform a multiobjective analysis and a complete description of methods can be found in ${ }^{(19,20,21)}$. This paper, the linear scalairisation method has been selected. It consists in merging two cost functions into one by adding linear weights to each function. Here, we have:

$$
f_{0}(x)=\alpha_{1} f_{1}(x)+\alpha_{2} f_{2}(x)
$$

Where $f_{0}(x)$ is the merged cost function , $\alpha_{1}$ the weight of the first cost function and $\alpha_{2}$ the weight of the second cost function.

Finally, the multiobjective procedure consists in minimizing $f_{0}(x)$ for different values of $\alpha_{1}$ and $\alpha_{2}$. When $\alpha_{1}$ is negligible in comparison with $\alpha_{2}$, the multiobjective optimization will be equivalent to the sole minimization of $f_{2}(x)$ and when $\alpha_{2}$ is negligible compared to $\alpha_{1}$, the multiobjective optimization will be equivalent to the sole minimization of $f_{1}(x)$. Then, by varying $\alpha_{1}$ and $\alpha_{2}$, a complete set of different but optimal solutions will be found. It is important to note that the multiobjective optimization procedure used in this paper is sensitive to the $\alpha_{1} / \alpha_{2}$ ratio. Then, it has been decided to fix the value of $\alpha_{1}$ to 1 and change the value of $\alpha_{2}$. The values of $\alpha_{2}$ have been empirically chosen in order to have the widest range of solution

\subsection{Cost functions: Travel time and fuel use}

In this paper, two cost functions are considered :

- $f_{1}(x)$ that is the total travel time on the chunk

- $f_{2}(x)$ that is the energy use (fuel use) on the chunk While $f_{1}(x)$ is easy to estimate from a generated speed profile, $f_{2}(x)$ can be hard to estimate. Then, a fuel use modelling, called SEFUM (Semi-Empirical Fuel Use Modeling), developed and validated in (8) has been used.

The basic hypothesis of SEFUM is that the tank-to-wheel efficiency ratio can be estimated from a polynomial function of the vehicle speed empirically measured for all vehicles. This is a complete analytical model, in opposition to states models, that should result in low computation times. The innovative idea is then to consider that one equation can represent all vehicle states. Therefore, the analytical developments are consistent with an automatic gearbox vehicle but it is assumed that the model will be efficient for manual gearbox vehicles even if engine speed is not directly taken into account. The theoretical consumed energy, $\mathrm{E}_{\text {theo }}$, in a time step dt is evaluated by the following formulae:

$$
E_{\text {theo }}=\left(\frac{1}{2} \rho_{a i r} S C_{x} v^{2}+C_{r r} m g+m p g+m a\right) v d t
$$

where $\rho_{\text {air }}=1.2 \mathrm{~kg} \mathrm{~m}^{-3}$ is the density of the air, $S\left(\mathrm{~m}^{2}\right)$ is the end face, $C_{x}$ is the longitudinal drag coefficient, $C_{r r}=0.015$ is the coefficient of rolling resistance, $m(\mathrm{~kg})$ is the vehicle mass, $g=9.81 \mathrm{~m} \mathrm{~s}^{-2}$ is the standard gravity, $\mathrm{p}$ (\%) is the road grade, $a\left(m s^{-2}\right)$ is the vehicle longitudinal acceleration, $v$ the vehicle longitudinal speed and $d t(s)$ the time step. 
Then, the efficiency ratio has been applied to take into account the energy losses in the combustion process and transmission. This ratio has been deduced from real experiments from Wang et al. (2008)(9) where the fuel consumption has been measured at different speeds. This method assumes that the engine speed can be neglected.

$$
\eta=\frac{E_{\text {theo }}}{E_{\text {meas }}}=10^{5} \frac{\frac{1}{2} \rho_{\text {air }} S C_{x} v^{2}+C_{r r} m g+m p g}{f(v) e_{\text {carb }} \rho_{\text {carb }}},
$$

where $E_{\text {theo }}$ is the theoretical energy consumed if the vehicle was the one tested by Wang et al. (9), $E_{\text {meas }}$ the measured energy consumed by the test vehicle, $f(v)=0.00026 v^{4}-0.018 v^{3}+$ $0.48 v^{2}-5.5 v^{2}+25$ is a function fitted from the work of Wang et al. which gives the fuel consumption in liters per hundred kilometers according to the vehicle speed, $e_{\text {carb }}=42.5 \times$ $10^{6} \mathrm{~J} \mathrm{~kg}^{-1}$ is the energy density of fuel and $\rho_{\text {carb }}=$ $0.76 \mathrm{~kg} \mathrm{~L}^{-1}$ is the fuel density.

\subsection{Optimization algorithm}

The selected algorithm is the simulated annealing(7). This algorithm simulates the annealing process for hardening steel. The main idea of this algorithm is to define an initial temperature and to reduce it at each iteration. An initial solution $x$ is evaluated through the $f_{0}(x)$ cost function. Then, a random move, whose the amplitude depends on the current temperature, is evaluated. If the new position is better than the previous one, it is accepted. Otherwise, the solution is accepted with a probability depending on the temperature.

Here, $x$ is a vector of five elements describing the speed profile of one chunk:

- $\quad x_{1}$, a: Initial acceleration $\left(\mathrm{ms}^{-2}\right)$

- $\quad x_{2}, \mathrm{~V}_{\text {des}}$ : desired speed $\left(\mathrm{ms}^{-1}\right)$

- $x_{3}, \mathrm{p}_{\mathrm{a}}$ : percentage of distance of acceleration phase

- $x_{4}, \mathrm{p}_{\mathrm{d}}$ : percentage of distance of deceleration phase

- $\quad x_{5}, \mathrm{~d}$ : Final deceleration $\left(\mathrm{ms}^{-2}\right)$

The minimum, maximum and initial values of these elements are given in table 1 . These values have been selected in order to represent the maximum physical range. Figure 4 shows the sensitivity of the algorithm to the initial values.

Table 1: Minimum, maximum and initial values of solution elements: $\mathrm{V}_{\max }$ is the maximum speed on the considered chunk

\begin{tabular}{|l|c|c|c|}
\hline Element & $\begin{array}{l}\text { Minimum } \\
\text { value }\end{array}$ & $\begin{array}{l}\text { Maximum } \\
\text { value }\end{array}$ & Initial value \\
\hline$\left.x_{1},{\mathrm{a}\left(\mathrm{ms}^{-2}\right)}^{-1}\right)$ & 0.2 & 8 & 3 \\
\hline$x_{2}, \mathrm{~V}_{\mathrm{des}}\left(\mathrm{ms}^{-1}\right)$ & $0.25 \mathrm{~V}_{\max }$ & $\mathrm{V}_{\max }$ & $0.75 \mathrm{~V}_{\max }$ \\
\hline$\left.x_{3}, \mathrm{~Pa}_{\mathrm{a}} \%\right)$ & 10 & 33 & 17 \\
\hline$x_{4}, \mathrm{P}_{\mathrm{d}(\%)}$ & 10 & 33 & 17 \\
\hline$x_{5}, \mathrm{~d}\left(\mathrm{~ms}^{-2}\right)$ & -0.2 & -8 & -2 \\
\hline
\end{tabular}

\section{Experimental Dataset description}

To evaluate the performances of the algorithm, it has to be compared to real experiments. This has been done using an experimental dataset that is hereafter described.

The experiments were performed with two classes of driving behaviors on the same route: "normal" and fuel efficient way to drive commonly known as "ecodriving”. Twenty-one drivers participated in these experiments that took place in June and July 2009 in Jouars-Pontchartrain (Yvelines) in France. In order to minimize traffic influence, the chosen route is of interurban type with a length of $15 \mathrm{~km}$. The trips were all performed under free flow conditions and with dry weather. First of all, the journey is discovered by the subjects while seeing the observer driving and giving safety and direction instructions. Then, the trip was driven twice by each driver: Firstly with a normal driving, and secondly following the "Golden Rules" of eco-driving defined and used in the Ecodrive project (Ecodrive, 2009):

- Change Gear upwards as soon as possible: Shift up between 1.500 and 2.000 revolutions.

- Do not accelerate quickly or harshly.

- Anticipate traffic flow: Look ahead as far as possible and anticipate what is happening. Plan your actions accordingly. Do not brake sharply or heavily.

- Maintain a steady speed at low RPM: Use the highest gear for the conditions and drive with low engine speed.

- Decelerate Smoothly: When you have to slow down or to stop, decelerate smoothly and leave the car in gear as long as is possible.

These rules were given in a written form to be read by the drivers just before the ecological trip. To eliminate a learning effect of the journey, trips order has been counterbalanced.

\subsection{Test site road description}

Experimental data have been acquired on road containing interurban and urban networks in the region of Paris in France. This road has been selected to represent a wide variety of travels on various road characteristics (road grade, curvature,. . .). The trip is $15 \mathrm{~km}$ long. Road parameters have been measured using a dedicated instrumented vehicle, called VANI (Vehicle for ANalysis of Itineraries). This vehicle has been validated over a large range of roads in France. For instance, an important measurement is the road slope, as presented in Fig. 3. This figure presents how the slope varied along the trip and this information is taken into account in every fuel use modeling tested in this study. In the case of this route, the slope varies approximately from $-5 \%$ to $5 \%$ which represents a hilly road in France. Very steep slopes, as mountain roads, have not been taken into account in this study.

\subsection{Test vehicle}

The vehicle used was a petrol-driven Renault Clio III with a 5 gears manual gearbox from 2008 which is representative of the French private passenger car fleet. The vehicle has been equipped with a VECTOR box CAN datalogger directly connected to a Personal Computer with the Windows OS. Data are collected through RTMaps software at a $20 \mathrm{~Hz}$ frequency. Fuel measurement reference is obtain from the CAN signal at a 80 $\mathrm{mm} 3$ resolution. The other logged signals involved the vehicle longitudinal speed, acceleration, RPM and gas pedal position. The vehicle has been also equipped with a GPS, a camera in front of the vehicle and a fuel flow meter DFL1 $\mathrm{x}$-5bar used to validate the fuel consumption estimated from the CAN bus.

\subsection{Test participants}

21 test participants have been recruited. Four of these drivers were ecodriving instructors while others were recruited among one thousand persons working in two different research institutes. They were asked to drive in two conditions: normal driving (no modification of their driving style) and ecodriving (after a short theoretical training as defined in Barkenbus $(2010)^{(22)}$, Barth and Boriboonsomsin $(2009)^{(23)}$, and Beusen et al. (2009) ${ }^{(24)}$ ). For ecodriving conditions, advice have been given to the drivers: do not accelerate too strongly, shift gear as soon as possible, 
anticipate traffic. These rules, whose some of them are yet under discussion, are considered as a definition of ecodriving at the time of the study. The use of two conditions for the experimental data has been helpful in the evaluation of fuel use model. Actually, fuel use model, and more specifically backward models, are sensitive to the driving patterns. This is due to the efficiency functions that always simplifies the real efficiency.

\section{Evaluation of the trajectory planning algorithm}

The performed evaluation is twofolds: Firstly a convergence and sensitivity of the simulated annealing is necessary to warrant the optimality of the proposed method. Secondly, results are compared on the dataset test route using the Pareto frontier and dominancy definitions.

\subsection{Results of simulated annealing}

The first step has been to evaluate the convergence of the simulated annealing on a specific condition. Here, it has been selected to work on the minimization of travel time only, on a 400 meters chunk with a speed limit of $25 \mathrm{~ms}-1$. In these conditions, the minimum travel time, considering an acceleration and deceleration norm limited to $8 \mathrm{~ms}-2$, is 19.1 seconds. This time is underestimated as acceleration is not constant in the speed profile generator. Figure 3 shows a simulation run. It can be seen that the convergence is reached in a few iterations (less than 500) with a value under 20 seconds which is close to the optimal value. This convergence can be reached on any test in 500 iterations.

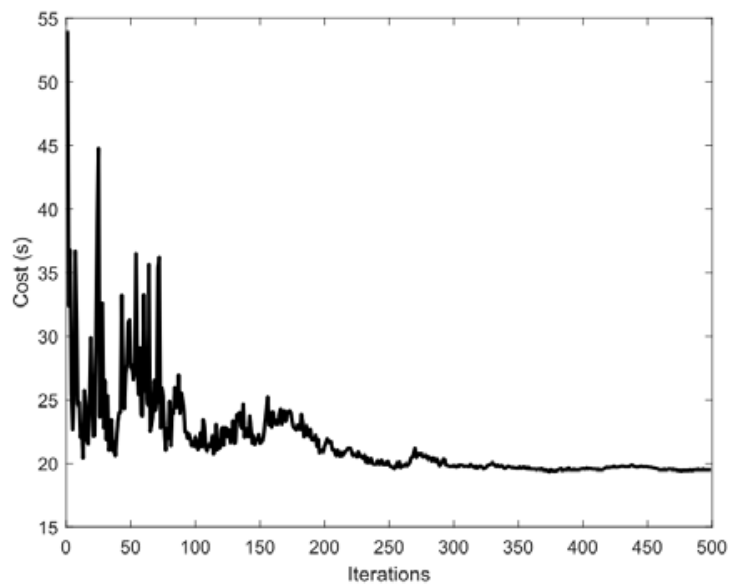

Fig. 3: Convergence of simulated annealing optimization on a B type chunk of 400 meters limited at $25 \mathrm{~m} / \mathrm{s}$

The second step consisted in providing different initial values of the variables to the simulated annealing. Then, the five elements of the solution a, Vdes, pa, pd and d have been modified from their lower limit to their upper limit with ten replications for each tested value as simulated annealing has a stochastic behavior. Ten values have been tested for each variable which makes 100 simulations per variable. The final travel time, after 500 iterations, is then plotted using boxplots in Figure 4. It can be seen that there is almost no variability due to the strong variations of the initial values of the algorithm as travel time varyies between 19.5 and 20 $\mathrm{s}$ (medians between 19.65 and 19.8).

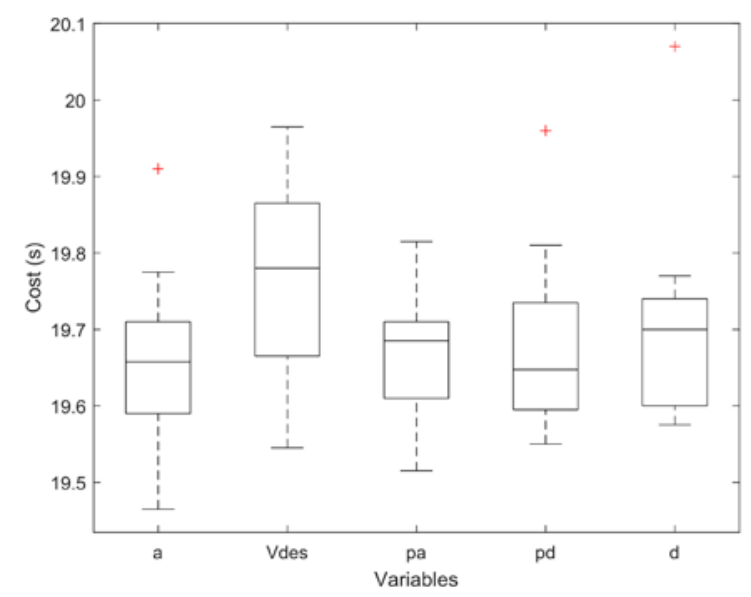

Fig. 4: Sensitivity of simulated annealing on the initial values of optimized variables

Simulated annealing is famous for being hard to calibrate. Then, the third step consisted in testing the influence of two parameters of the simulated annealing in order help in the calibration procedure. The first one is the initial temperature that is well known for having a strong effect on results. The second one is the temperature decreasing ratio that is assumed to have an influence on the minimization quality. These two parameters have been tested between a lower and upper bound. 7 values have been tested for the temperature decreasing ratio and 8 values for the initial temperature. In this case, all combinations of parameters have been tested 10 times and averaged. Results are displayed in Figure 5. In this figure, it can be noticed that higher the temperature is and higher is the travel time and worse is the convergence. On the other hand, higher the temperature decreasing rate is and better is the convergence. However, for decreasing rates closer to 1 , the convergence is worse as the algorithm become as efficient as a purely random algorithm.

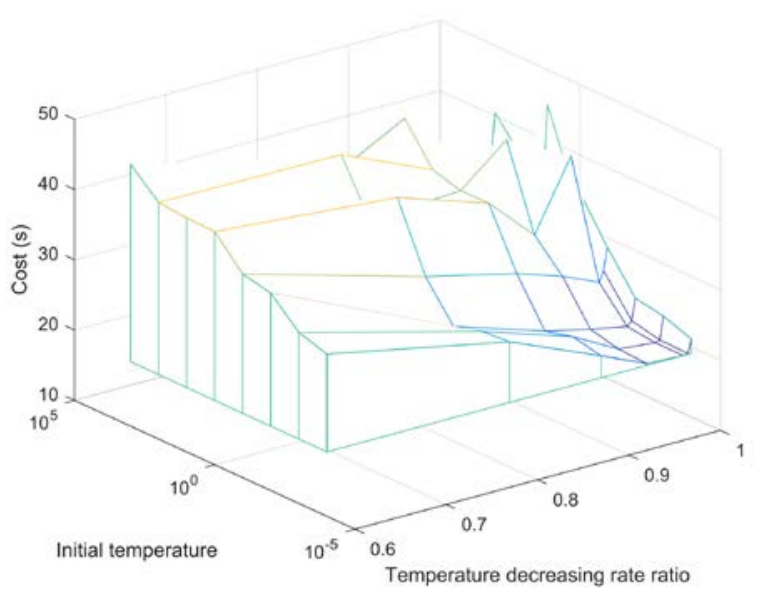

Fig. 5: Estimation of convergence of simulated annealing under the influence of initial temperature and temperature decreasing ratio 


\subsection{Global evaluation}

A global evaluation has then been performed by comparing the results of the whole algorithm to experimental results and results from another multi-objective optimization method (Dijkstra's), described in the following sub section.

\subsubsection{Dijkstra’s multi-objective optimization}

Dijkstra's algorithm is an algorithm for finding the shortest paths between nodes in a graph ${ }^{(25)}$. The algorithm used in this paper is a modified version of the $\mathrm{A}^{*}$ presented in ${ }^{(26)}$ without heuristics. The optimization conditions has been set to the same values as the MOSA and both algorithms have been implemented under Matlab software. A notable difference between the two algorithms is that Dijkstra's method computes optimal speed at a fixed distance step of 10 meters where MOSA computes at a fixed time step of 0.05 sesond. Thus Dijkstra's algorithm can test more speed possibilities because speed is not constrained by a function but at lower frequency. This difference is due to the nature of the two algorithms Dijkstra's being a graph search algorithm and MOSA a piecewise continuous functions optimization method.

\subsubsection{Results}

First, Figure 6 shows optimized speed profiles on the same route as where experiments have been performed. Results show strong differences between the speed profile in the extremum conditions. The specificity of the chunking method shows some of its limits because transition points necessarily needs to be reached while humans would probably not drive this way. Total computation time is around 300 seconds which is widely enough for real time computation at $50 \mathrm{~ms}-1$.

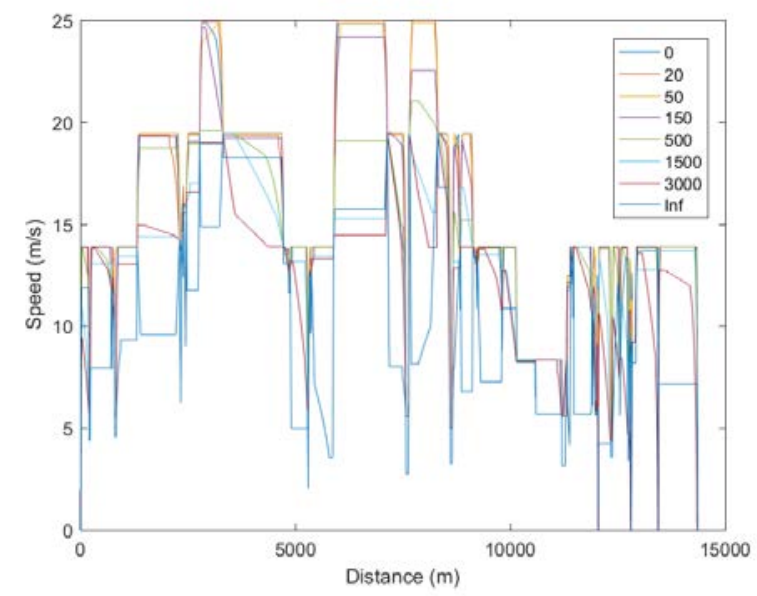

Fig. 6: Generated speed profiles where the weight on fuel use $\alpha_{2}$ varies from 0 to infinite. The legend lists the $\alpha_{2}$ values

Finally, it is important to compare global results to experimental and other numerical data. In figure 7, results from several speed profiles are plotted in 2D graph where each axis represent an objective of the problem. According to the Pareto definition of dominancy, the proposed algorithm (MOSA) outperforms the
Dijkstra's method as travel time and fuel use are always under the Dijkstra's ones. However, it cannot find a solution with a lower travel time. It can also be seen that some drivers did better than the algorithm could find. By analyzing data, it is probably due to the fact that human drivers have overspeeded in some parts of the road where the algorithms strictly observed the driving laws.

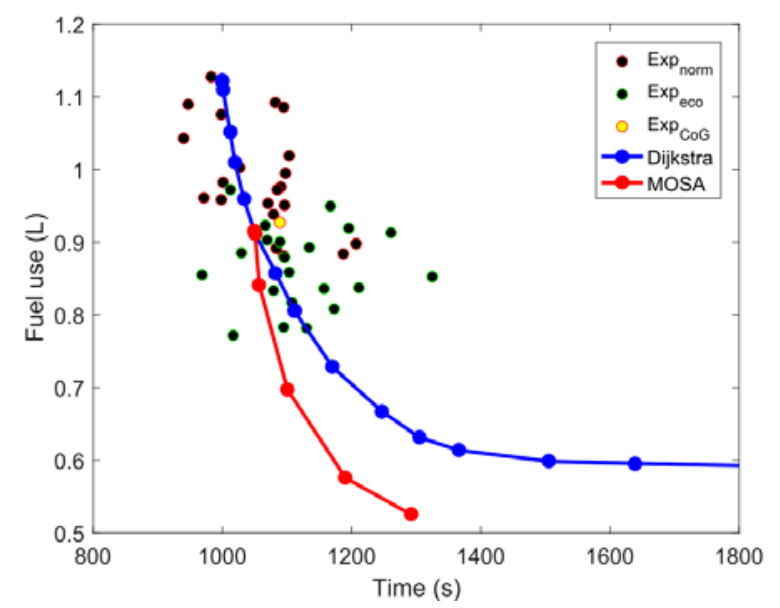

Fig. 7: Experimental and numerical Pareto plots.

Figure 8 shows an example optimal solution on one chunk versus the variation of the $\alpha_{2}$ coefficient.

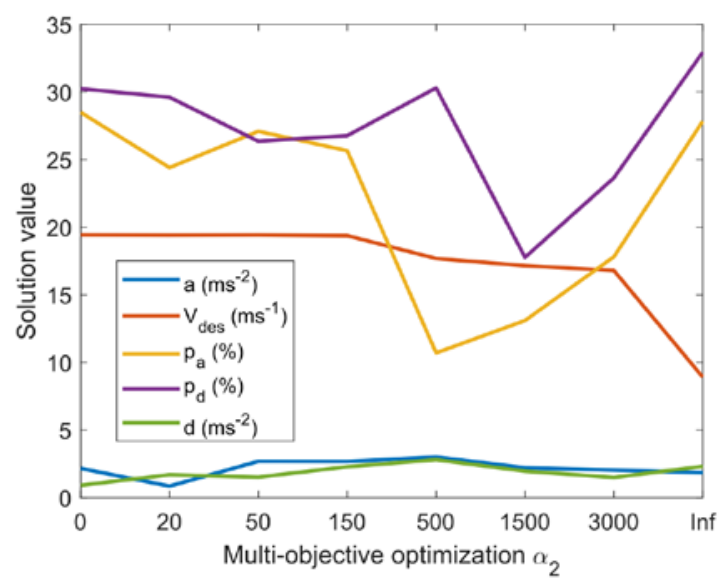

Fig. 8: Experimental and numerical Pareto plots.

\section{Discussions}

Results show that this speed profile planning method is able to generate rapidly (real time) optimal speed profiles that comply with safety constraints and that can reduce energy use compared to experimental results on driving simulator without trading-off travel time.

However several limitations need to be taken into account:

- $\quad$ No preceding vehicle or Vulnerable Road User have been included into these simulations. The algorithm needs to be complemented by a more reactive method that will act in real time when obstacles appear in front of the vehicle.

- The chunking method reduces the quality of optimization as nothing guarantees that the sum of optimal chunk will provide an optimal speed profile. This can be counter balanced as the road is rarely totally free of other vehicles that will induce a speed change. 


\section{Olivier Orfila et al. / International Journal of Automotive Engineering}

Vol.10, No.1 (2019) pp.26-33

- The sensitivity analysis of simulated annealing has not been performed on all possible combinations due to computation time constraints.

- The linear scalairisation method is not able to build a Pareto frontier in a non-convex optimization problem. However, the convex properties of the optimization have not been tested. In this specific case, the multiobjective optimization can be done using epsilonconstraint method using results of this paper to check if the problem is convex or not.

- The comparison with experiments is limited due to the human drivers that did not follow driving rules.

\section{Conclusion}

This paper presented a planning algorithm able to produce a multi-objective optimization of a speed profile in real-time. Fuel use and travel-time are optimized while constraints have been defined based on safety aspects.

Results show that the proposed method outperforms the classical Dijkstra's algorithm in terms of convergence. Human drivers did better than the algorithm on the travel time (because of overspeeding) while they were worse on the fuel use objective.

The method used for optimization, the simulated annealing, has been completely calibrated and tested using a sensitivity analysis. This analysis proved that the algorithm is robust to any variation in the initial values of variables to be optimized.

Future works will concentrate on determining the weight of criteria for the optimization procedure according to the driver preferences. A first implementation could be that the driver choose the level through HMI but a smarter solution would be to learn directly from the driver habits using machine learning when the driver is driving the car.

Driving tests have been performed with the agreement of IFSTTAR ethics committee.

This paper is written based on a proceedings presented at JSAE FAST-zero'17 Meeting.

\section{References}

(1) David michael stavens Learning to drive: Perception for autonomous cars. Dissertation submitted to the department of computer scienceand the committee on graduate studies of stanford university (2011)

(2) B Paden, M Cap, SZ Yong, D Yershov, E Frazzoli : A Survey of Motion Planning and Control Techniques for SelfDriving Urban Vehicles IEEE Transactions on Intelligent Vehicles Vol. 1 No. 1, pp. 33-55 (2016)

(3) Christos Katrakazas, Mohammed Quddus, Wen-Hua Chen, Lipika Deka, Real-time motion planning methods for autonomous on-road driving: State-of-the-art and future research directions, Transportation Research Part C: Emerging Technologies, Vol. 60, pp. 416-442 (2015) ISSN 0968-090X, https://doi.org/10.1016/j.trc.2015.09.011.(Accessed. 2019.01.07)

(4) Steven M. LaValle, Planning algorithms, Copyright 2006, Cambridge University Press (2006)

(5) Kung, H. T.; Luccio, F.; Preparata, F.P. "On finding the maxima of a set of vectors.". Journal of the ACM. Vol. 22, No. 4, pp. 469-76. doi:10.1145/321906.321910, (1975)
(6) Kalyanmoy Deb, Multi-objective optimization using evolutionnary algorithms, Wiley, ISBN 0-471-87339-X, (2002)

(7) Kirkpatrick, S.; Gelatt Jr, C. D.; Vecchi, M. P. "Optimization by Simulated Annealing". Science. Vol. 220, No. 4598, pp. 671680. doi:10.1126/science.220.4598.671. (1983)

(8) O. Orfila, C. Freitas Salgeiredo, G. Saint Pierre, H. Sun, Y. Li, D. Gruyer, S. Glaser, Fast computing and approximate fuel consumption modeling for Internal Combustion Engine passenger cars. Article in Transportation Research Part D Transport and Environment Vol. 50, pp. 14-25, (2017)

(9) Haikun Wang, Lixin Fu, Yu Zhou, He Li, Modelling of the fuel consumption for passenger cars regarding driving characteristics, Transportation Research Part D: Transport and Environment, Vol. 13, No. 7, pp. 479-482, ISSN 1361-9209, http://dx.doi.org/10.1016/j.trd.2008.09.002.(Accessed.

2019.01.07)

(10) S. Sivaraman and M. M. Trivedi, "Looking at Vehicles on the Road: A Survey of Vision-Based Vehicle Detection, Tracking, and Behavior Analysis,” IEEE Trans. Intell. Transp. Syst., Vol. 14, No. 4, pp. 1773-1795, (2013)

(11) D. Gerónimo, A. M. López, A. D. Sappa, and T. Graf, "Survey of Pedestrian Detection for Advanced Driver Assistance Systems,” IEEE Trans. Pattern Anal. Mach. Intell., Vol. 32, No. 7, pp. 1239-1258, (2010)

(12) Hao Zhu, Ka-Veng Yuen, Lyudmila Mihaylova and Henry Leung, "Overview of Environment Perception for Intelligent Vehicles.”, IEEE Transactions on Intelligent Transportation Systems. ISSN 1524-9050 (2017)

(13) B. Vanholme, D. Gruyer, B. Lusetti, S. Glaser, S. Mammar, "Highly automated driving on highways based on legal safety", in IEEE Transaction on Intelligent Transportation Systems, Vol 14, No. 1, pp 333-347, (2013)

(14) Paden B., Čap M., Yong S. Z., et al. A Survey of Motion Planning and Control Techniques for Self-driving Urban Vehicles. In IEEE Transactions on Intelligent Vehicles, Vol. 1, No 1, pp. 33-55, (2016)

(15) Bevly D., Cao X., Gordon, M., et al. Lane Change and Merge Maneuvers for Connected and Automated Vehicles: A Survey. In IEEE Transactions on Intelligent Vehicles, Vol. 1, No 1, pp. 105120, (2016)

(16) González D., Pérez J., Milanés V., Nashashibi F.. A review of motion planning techniques for automated vehicles. In IEEE Transactions on Intelligent Transportation Systems, Vol. 17, No 4, pp. 1135-1145, (2016)

(17) Martin Treiber, Ansgar Hennecke, Dirk Helbing, “Congested Traffic States in Empirical Observations and Microscopic Simulations”, Physical Review E, Vol. 62, pp. 1805-1824, (2000)

(18) Treiber, Martin, Kesting, Arne, "Traffic Flow Dynamics Data, Models and Simulation”, Springer edition, ISBN 978-3642-32460-4 (2013)

(19) K. Deb. Multi-Objective Optimization using Evolutionary Algorithms, Wiley-Interscience Series in Systems and Optimization. John Wiley \& Sons, Chichester (2001) 
(20) E. Zitzler, K. Deb, and L. Thiele. Comparison of Multiobjective Evolutionary Algorithms: Empirical Results. Evolutionary Computation, Vol. 8, No. 2, pp.173-195 (2000)

(21) Jürgen Branke, Kalyanmoy Deb, Kaisa Miettinen, and Ralph E. Steuer, editors. Practical Approaches to Multi-Objective Optimization, 7.-12. November 2004, volume 04461 of Dagstuhl Seminar Proceedings, 2005. Internationales Begegnungs- und Forschungszentrum (IBFI), Schloss Dagstuhl, Germany IBFI, Schloss Dagstuhl, Germany. (2005)

(22) Jack N. Barkenbus, "Eco-driving: An overlooked climate change initiative", in Energy Policy, the International Journal of the Political, Economic, Planning, Environmental and Social Aspects of Energy, Elsevier, Vol. 38, pp. 762-769 (2010)

(23) M. Barth, K. BoriboonsomsinEnergy and emissions impacts of a freeway-based dynamic eco-driving systemsTransportation Research Part D: Transport and Environment, Vol 14., No 6, pp. 400-410 (2009)

(24) B. Beusen, S. Broekx, T. Denys, C. Beckx, B. Degraeuwe, M. Gijsbers, K. Scheepers, L. Govaerts, R. Torfs, and L. Int Panis, "Using on-board logging devices to study the longer-term impact of an eco-driving course," Transportation Research Part D : Transport and Environment, Vol. 14, No. 17, pp. 514-520 (2009)

(25) Cormen, Thomas H.; Leiserson, Charles E.; Rivest, Ronald L.; Stein, Clifford "Section 24.3: Dijkstra's algorithm". Introduction to Algorithms (Second ed.). MIT Press and McGraw-Hill. pp. 595-601 (2001). ISBN 0-262-03293-7

(26) Sébastien Glaser, Olivier Orfila, Roman Potarusov, Lydie Nouvelière, Speed profile optimization for electric vehicle with regenerative capacity, FAST-Zero'2013 Proceedings, No..OS-1-1 (2013) 